\title{
La recherche, affaire d'État. Enjeux et luttes d'une politique fédérale des sciences
}

Martin Benninghoff et Jean-Philippe Leresche. Presses polytechniques et universitaires romandes, coll. Le savoir suisse, Lausanne, 2003, $134 \mathrm{p}$.

\section{Muriel Le Roux}

\section{(2) OpenEdition}

\section{Journals}

Édition électronique

URL : https://journals.openedition.org/histoire-cnrs/426

DOI : $10.4000 /$ histoire-cnrs. 426

ISSN : 1955-2408

Éditeur

CNRS Éditions

Édition imprimée

Date de publication : 5 mai 2004

ISBN : 978-2-271-06145-4

ISSN : $1298-9800$

\section{Référence électronique}

Muriel Le Roux, "La recherche, affaire d'État. Enjeux et luttes d'une politique fédérale des sciences », La revue pour l'histoire du CNRS [En ligne], 10 | 2004, mis en ligne le 07 mars 2006, consulté le 20 mai 2021. URL : http://journals.openedition.org/histoire-cnrs/426 ; DOI : https://doi.org/10.4000/histoirecnrs.426

Ce document a été généré automatiquement le 20 mai 2021.

Comité pour l'histoire du CNRS 


\section{La recherche, affaire d'État. Enjeux et luttes d'une politique fédérale des sciences}

Martin Benninghoff et Jean-Philippe Leresche. Presses polytechniques et universitaires romandes, coll. Le savoir suisse, Lausanne, 2003, 134 p.

\section{Muriel Le Roux}

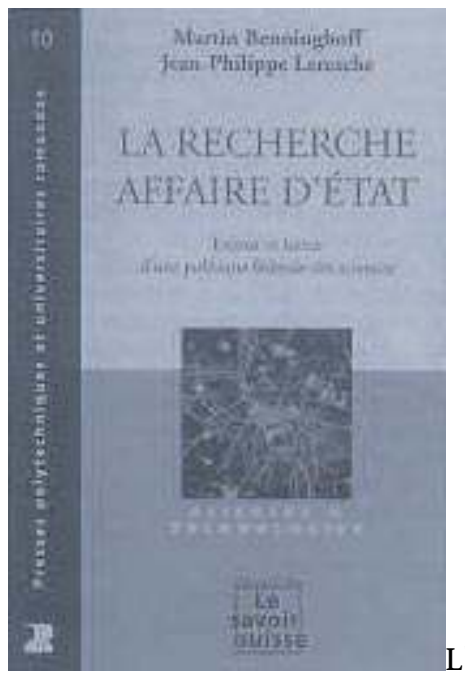

L'ouvrage de Martin Benninghoff et Jean-Philippe Leresche est intéressant à plus d'un titre. C'est un livre de vulgarisation, sans prétention. Mais comme cela arrive souvent, lorsque ce type d'ouvrage est parfaitement maîtrisé par les auteurs, il est d'une remarquable efficacité. En 134 pages, les auteurs présentent le couple "infernal »: État-recherche en Suisse des années 1930 à nos jours et traitent des enjeux de la genèse de la politique fédérale de la recherche au pays du fédéralisme.

Le regard des politologues sur l'histoire de la recherche apporte un éclairage sur le fonctionnement des rouages institutionnels, préalable nécessaire à l'étude, par les autres disciplines des sciences humaines et sociales, du monde de la recherche. Car en 
Suisse comme en France, la recherche est "une affaire d'État ». Décrivant le présent dans un premier chapitre problématique, ils balaient le XIX ${ }^{e}$ siècle et rappellent que les années 1930 furent la période de gestation des premières institutions fédérales helvétiques dans le second chapitre. Les trois chapitres suivants sont consacrés à l'émergence d'une politique des sciences (1960-1970), la promotion d'une science utile (1970-1990) et à la gouvernance de la science (1990-2000). Ils concluent dans un dernier chapitre en s'interrogeant sur le devenir de la recherche.

2 Or, aujourd'hui, dans le cadre de l'Union européenne, les gouvernements des pays membres sont tenus d'harmoniser leurs pratiques administratives, industrielles et commerciales, donnant lieu à de nombreuses analyses appelées le new public management. Cette nouvelle politique de gestion publique touche aussi bien les administrations que les entreprises publiques. En Suisse, tout comme en France, certains secteurs ont été soustraits à la gestion de l'État (télécoms, transports, énergie en Suisse, Rhône-Poulenc, Pechiney, etc., en France) ou sont en voie de privatisation (les Postes et le gaz en Suisse, France Telecom). Or la recherche, malgré des débats réguliers à son sujet, n'a jamais été soustraite au giron de l'État dans ces deux pays. Ce fait est d'autant plus important que depuis le tournant des années 2000, et spécifiquement depuis la conférence de Lisbonne de mars 2000, les États membres de l'Union se sont fixés comme objectif d'accroître les dépenses de R\&D afin d'atteindre $3 \%$ du PIB, 2/3 devant être financés par le secteur privé. L'enjeu est la construction d'un espace européen de la recherche capable de concurrencer la recherche américaine.

3 Alors que les Suisses avaient très majoritairement refusé de rejoindre l'Union européenne en 1992, ce livre nous montre qu'en revanche, la Confédération avait déjà depuis quelques années accepté de participer aux programmes européens de recherche. Il s'agissait de rester compétitif et attractif au sein de cette économie de la connaissance qui offre un nouveau statut à la science. L'attrait pour la connaissance en soi est bien moindre, c'est devenu un bien économique, un facteur de croissance. Dans ce contexte, le rôle qui incombe à l'État en matière de politique scientifique et de promotion de la recherche est important, notamment lorsqu'il s'agit de son organisation ou de sa finalité. Les auteurs rappellent qu'en Suisse pas plus qu'ailleurs la recherche fondamentale et libre n'a pas été supplantée par la recherche orientée et/ou sur commande ou sous contrat. Il ressort que, là encore, la Confédération, depuis plus de vingt ans, connaît des mouvements de balanciers, l'État fédéral soutenant tantôt l'une ou tantôt l'autre. Rien de bien original, que ce soit les programmes prioritaires de recherche et les pôles nationaux de recherche helvétiques en regard des actions concertées incitatives françaises... Force est de constater toutefois que l'intensité et la rapidité de la réforme varient d'un pays à l'autre. En France, la politique de la recherche se définit par son inertie, ce qui fait dire à nos auteurs que notre système privilégie les positions acquises, que sa bureaucratie est centralisée et opaque... Plus grave, la comparaison des cas français et suisse confirme ce que beaucoup de chercheurs français savent et que bien peu évoquent dans le cadre de discussions pour un changement concerté : à savoir que les réformes sont rares et superficielles, que bien peu d'organismes de recherche usés disparaissent, que les évolutions sont lentes et de petite envergure. Lorsqu'elles ont lieu, elles privilégient la création de nouvelles institutions. Les évaluations de certains programmes existent, mais la politique générale n'est jamais évaluée et l'on se demande même si elle est véritablement pilotée. Pour situer la Suisse dans l'espace international de la science, les références à la France 
sont fréquentes, les auteurs étant francophones, mais les comparaisons avec l'Allemagne, les Pays-Bas, la Belgique et les États-Unis le sont tout autant. D'ailleurs, il en ressort que la Suisse n'échappe pas aux tendances générales qui caractérisent la recherche européenne.

En revanche, ce qui la différencie de la France, c'est la pratique de la démocratie directe du fédéralisme et le respect de l'autonomie des lieux académiques. La recherche publique est financée par le secteur privé à concurrence de $70 \%$ de ses budgets. Jusqu'à la dernière période, le système de recherche suisse était complexe, sans véritable pouvoir central et dépendait d'un très grand nombre d'organismes. La recherche est gérée selon le système de la concordance qui en d'autres termes est une démocratie politique consensuelle. La stabilité politique de la Confédération (récemment remise en cause) autorisait cette gestion démocratique de la recherche. Le fédéralisme qui est la pratique politique du compromis entre le gouvernement fédéral et les cantons évolue en fonction des rapports de forces. La démocratie directe, très liée au système de concordance, se traduit tout au long du second $\mathrm{XX}^{\mathrm{e}}$ siècle par un libéralisme politique et une faible bureaucratie en matière de politique de recherche. La création du Fonds national de la recherche scientifique après la Seconde Guerre mondiale devait permettre de réduire le retard qui existe entre la Suisse et les États-Unis. Cette reconstruction de la recherche suisse ne s'est pas faite selon le modèle français trop centralisé, ni selon le modèle allemand trop décentralisé. Le but était de soutenir de façon équitable l'ensemble des disciplines et de réduire les disparités entre la recherche cantonale et celle des écoles polytechniques fédérales. Toutes les émanations de la société suisse étaient représentées selon différentes instances. Les scientifiques sont consultés pour les orientations à suivre, tout comme les citoyens. La science est un enjeu de société car jusqu'aux années 1960, elle participe à la construction de l'identité nationale. Donc le politique se retrouve dans la façon dont les projets sont élaborés et influe aussi bien le FNRS que le Conseil de la recherche.

5 Tout comme en France, la rupture eut lieu au cours des années 1970. Sous la pression des partis bourgeois, la recherche libre est remise en cause : on demande à la recherche des justifications et de la rentabilité. La crise économique et bientôt les grandes pollutions remirent en cause le pouvoir de la science dans la construction du futur. Les cantons prirent la main au détriment du pouvoir fédéral. Cela se traduisit par de nombreuses critiques, alimentées également par les rapports de l'OCDE, qui débouchèrent sur la réforme des institutions: les programmes de recherche d'intérêt général devant aboutir en cinq ans. Au terme de cette période, l'État fédéral devait aussi bien promouvoir la recherche libre et fondamentale que la recherche orientée ; il n'hésitait plus à intervenir aussi bien en matière de programme que de budget. C'est le triomphe de la R\&D (expression qui devient couramment employée à partir de 1974 partout en Europe) telle que définie par l'OCDE.

6 Au cours de la période 1990-2000, le Fonds national de la recherche scientifique réagit à ce qu'il considère comme une instrumentalisation par le politique. Pourtant, malgré sa résistance, le FNRS fut évalué tout comme les autres institutions financières, donnant naissance à des structures de réflexion sur ce que devait être la recherche; il fallait trouver un nouvel équilibre entre recherche fondamentale et recherche orientée, entre les instances fédérales de la recherche, les écoles polytechniques et les centres cantonaux. Le Conseil suisse de la science réalisa une contre-expertise par rapport à celle du FNRS, tandis qu'en 1996 était créé le poste de secrétaire d'État à la Recherche. 
Cela aboutira à la création des pôles nationaux de la recherche. Le projet reposant sur une large concertation avant d'être présenté au Parlement fut accepté sans difficulté. Toutefois, au cours de cette période, s'est aussi opérée une "politisation » de la politique de la recherche suisse, chaque acteur développant en son sein un organe afin de défendre son projet. Ainsi le FNRS doit être innovant pour conserver sa place dans la structure helvétique.

7 Les pratiques de la recherche sont différentes de celles qui prévalaient dans les années 1950 au cours desquelles la recherche était libre, la Confédération intervenant peu. Les années 1970 furent celles du partage entre recherche libre et recherche orientée. De 1980 à 1995, période de forte croissance, des allocations à la recherche permirent l'élaboration de structures complémentaires de concertation, d'élaboration des politiques et de gouvernance. La crise budgétaire perceptible dès 1995 va conduire l'État à réduire ses interventions. Tandis que la politique européenne influençait très largement la politique de recherche suisse, les fonds alloués par la Suisse à l'UE étaient déduits du budget fédéral. Il n'est désormais plus possible de faire abstraction du politique ; on voit le FNRS qui, à l'origine, se situait au-dessus des partis, jaloux de son indépendance, devenir un acteur de la politique parmi d'autres après en avoir intégré les pratiques.

Ce livre essentiel pour la compréhension des enjeux qui se jouent autour de la R\&D en Europe montre que les pratiques concertées permettent d'écrire une autre histoire de la politique de la recherche. Il est vrai que la taille de la Suisse induisant sa pratique du fédéralisme, de la démocratie l'a autorisée à créer et conduire une politique contractuelle et consensuelle tout au long de la période, très différente d'un schéma descendant à la française. Cela n'est pas sans intérêt au moment où justement le fédéralisme semble être un des possibles du futur européen.

9 En conclusion, même s'il apparaît qu'il n'est pas certain que les Suisses puissent continuer à gérer leur politique de la recherche comme ils l'ont fait jusqu'à présent du fait même de la construction de l'espace européen de la recherche, est-il besoin de rappeler l'urgence qui existe pour le milieu des chercheurs à entamer, en France, une réflexion sur ce que recouvre leur métier? Cette réflexion nous permettrait d'éviter que l'on se crispe sur des postions défensives, d'intégrer l'évolution du métier qui a bien changé depuis les débuts de sa professionnalisation. On s'apercevrait alors que notre milieu appartient à un collectif chargé du futur de la nation, voire de l'Europe... En un mot ne doit-on pas, comme le rappellent Martin Benninghoff et Jean-Philippe Leresche, investir plus de temps et d'argent dans la matière grise - tant nous portons en nous la capacité de changement -, les activités de recherche, dans les structures et infrastructures plutôt que dans la réforme institutionnelle ? Encore eut-il fallu qu'elles aient eu lieu. Contrairement à la Suisse, ce n'a pas été le cas de la France. 
AUTEUR

MURIEL LE ROUX

Chargée de recherche IHMC-CNRS-ENS 